\title{
Assessment of knowledge of junior doctors and non-specialists about musculoskeletal medicine
}

\author{
Saba Saif ${ }^{1}$, Samina Fida², Hala Mansoor ${ }^{3}$
}

\begin{abstract}
Objectives: To assess the knowledge and confidence of junior doctors and non-specialists in examining and making a diagnosis of patients with musculoskeletal (MSK) diseases.

Methods: This was a Cross-sectional study of 121 doctors working in medical clinics at a tertiary hospital between October and December 2019. Data were collected using a questionnaire. Doctor's awareness regarding different MSK examination methods including gait, arms, leg, spine (GALS), pediatric gait, arms, leg, spine (pGALS) and regional examination of musculoskeletal system (REMS) was noted. Undergraduate teaching of these methods and use in their daily practice was surveyed.

Results: Majority of the doctors lacked awareness about different MSK examination techniques. Awareness about GALS, REMS and pGALS was $44.6 \%, 59.5 \%$ and $18.2 \%$ respectively. There was significant correlation of GALS/REMS awareness with the undergraduate teaching and doctor's clinical experience ( $\mathrm{p}$-value $<0.05)$. Confidence level of doctors in diagnosing patients with adult MSK pathologies was 55\%. Only few doctors were satisfied with their musculoskeletal education (29\%).

Conclusion: The GALS examination is a useful screening tool for junior doctors and non-specialists in a direct access setting to rule out musculoskeletal problems.
\end{abstract}

KEYWORDS: Musculoskeletal, Physical Examinations, Musculoskeletal Diseases, Arthritis, Undergraduate Medical Education.

How to cite this:

doi: https://doi.org/10.12669/pjms.37.1.3148

Saif S, Fida S, Mansoor H. Assessment of knowledge of junior doctors and non-specialists about musculoskeletal medicine. Pak J Med Sci. 2021;37(1):175-179. doi: https://doi.org/10.12669/pjms.37.1.3148

This is an Open Access article distributed under the terms of the Creative Commons Attribution License (http://creativecommons.org/licenses/by/3.0), which permits unrestricted use, distribution, and reproduction in any medium, provided the original work is properly cited.

1. Dr. Saba Saif, FCPS. Rheumatology, FCPS Medicine. Assistant Professor,

2. Dr. Samina Fida, FCPS Medicine.

Associate Professor

3. Dr. Hala Mansoor, FCPS (Gastroentrol), FCPS Medicine, MRCP. Assistant Professor

1-3: Department of Medicine,

Division of Rheumatology Combined Military Hospital, Lahore, Pakistan.

Correspondence:

Dr. Saba Saif,

Department of Medicine,

Assistant Professor of Rheumatology,

Division of Rheumatology Combined Military Hospital,

Lahore, Pakistan.

Email: sabasajjadpk@gmail.com

* Received for Publication:

* $1^{\text {st }}$ Revision Received:

* $2^{\text {nd }}$ Revision Received:

* Final Revision Accepted:
April 30, 2020

June 30,2020

October 17, 2020

October 29, 2020

\section{INTRODUCTION}

Musculoskeletal (MSK) conditions affect millions of people around the world. About 10 million people in the UK are suffering from arthritis. ${ }^{1}$ MSK disorders are ranked first in prevalence as the cause of chronic health problems, long term disabilities, and consultations with a health professional. MSK disorders are underdiagnosed in patients with medical problems. MSK conditions affect almost one in five adults. ${ }^{2}$ Low back pain, osteoarthritis and soft tissue rheumatism are the main diagnosis. ${ }^{3}$ Chronic MSK pain can cause sleep disturbance, fatigue, low mood, limited activity and participation affecting individual's quality of life. ${ }^{4}$

Previously reported increases in undiagnosed cases of arthritis have highlighted the need for 
a simple yet sensitive screening MSK exam for identifying MSK abnormalities. Use of GALS should be considered as part of the assessment of all patients with joint problems. ${ }^{5}$ GALS is a simple and useful screening tool for routine MSK examination in hospitals and general practice and has been integrated into the undergraduate medical curriculum. Despite this there is evidence that doctors lack awareness and, competency in MSK examination and GALS is underperformed routinely. ${ }^{6}$ GALS provides a standardized approach to MSK examination while REMS is a more detailed examination of the relevant area, based on the 'look, feel, move' principle. Although being taught it is evident that GALS is under-utilized with $50 \%$ of clinicians not using GALS in their teaching. ${ }^{7}$ MSK examination skills can be improved by integrating peer assisted learning (PAL) into the undergraduate medical curriculum. ${ }^{8}$

The majority of causes of joint pains in childhood are benign, self-limiting and often traumarelated but can be the presenting features of malignancy, sepsis, vasculitis and non-accidental injury. ${ }^{9}$ The pGALS screen includes questions relating to pain and function but a negative response does not exclude a MSK disease, and therefore pGALS should be performed which on the average takes two minutes. ${ }^{10}$ To improve the confidence of junior medical officers in pediatric MSK assessment a clinical examination teaching intervention is required. ${ }^{11}$ Objective structured clinical examination (OSCE) based MSK workshop leads to significant improvement in knowledge and clinical skills among medical students and residents. ${ }^{12}$

This study was designed as joint pains are the most common presenting complaints in primary care settings, yet physicians don't know how to examine these patients. There is no emphasis on MSK examination in undergraduate and postgraduate teaching and the majority doctors find this difficult and challenging. Most of the patients remain undiagnosed at the primary health care level being treated by quacks, Hakeem's and other non-specialists till they develop irreversible deformities. Therefore, training the junior doctors will lead to earlier diagnosis, referral, treatment and prevention of permanent disability in such patients. The merits of introducing MSK examination into undergraduate curricula and practice should be explored. No local study was done before.

\section{METHODS}

This study was conducted in CMH Lahore, between October and December 2019, after approval from Institutional Review Board (Ref:437/ERC/CMHLMC, Dated 07-11-19). The sample size was calculated taking frequency of adequate MSK knowledge as 13\%; Confidence level $95 \%$, margin of error 6\%. Eligibility criteria was any doctor practicing in $\mathrm{CMH}$ and having a daily interaction (i.e. history taking and/or clinical examination) with patients. 121 subjects selected randomly from the OPD's and indoors voluntarily completed the survey. Subjects included mainly the junior doctors from different specialties (medicine, surgery and allied) and different non-specialists including General Practitioners and family physicians other than rheumatologists. Written informed consent was taken from each doctor and their confidentiality was maintained. Their demographic profiles (i.e. age, sex, designation, and department) and year/ place of graduation were noted using a structured questionnaire (annex-111).

The subjects were asked to mark their ability to diagnose/refer common MSK pathologies (on the basis of history and examination) in both adults and school-aged children on a 10 point scale. Questionnaire also inquired about the subject's awareness of different MSK examination methods i.e GALS, REMS and pGALS. Undergraduate teaching of these methods and use in their daily practice was also noted.

All the collected information was entered into SPSS version 22.0 and analyzed. Quantitative variables like age were presented as means with standard deviation. Qualitative variables like gender, specialty, designation, clinical experience and their confidence level in diagnosing MSK pathologies, were presented as frequency and percentages. Chi-square test of significance was applied to see significance of association of GALS, REMS, pGALS awareness with under-graduate teaching and clinical experience in years. P-value $\leq 0.05$ was considered statistically significant.

\section{RESULTS}

Total respondents were 121. Mean age of the subjects was 28.96 \pm 6.7 . Almost half the doctors were a graduate of $\mathrm{CMH}$ Lahore Medical College and the rest belonged to different medical colleges all over Punjab. The study included 39 house officers, 57 residents and 25 consultants. 
Table-I: Demographic Characteristics.

\begin{tabular}{lc}
\hline Demographics & $\begin{array}{c}\text { Frequency } \\
N(\%)\end{array}$ \\
\hline Female respondents & $71(58.7 \%)$ \\
Male respondents & $50(41.3 \%)$ \\
Time since primary medical & $74(61.2 \%)$ \\
$\quad$ qualification: <5 years & \\
Time since primary medical & $47(38.8 \%)$ \\
$\quad$ qualification: $>5$ years & \\
Current training specialty: & \\
Medicine & $45(37.2 \%)$ \\
Surgery & $13(10.7 \%)$ \\
General practitioner & $19(15.7 \%)$ \\
Orthopaedics & $12(9.9 \%)$ \\
Pediatrics & $13(10.7 \%)$ \\
Physiotherapists & $8(6.6 \%)$ \\
Family physician & $4(3.3 \%)$ \\
Other specialties & $7(5.8 \%)$ \\
\hline
\end{tabular}

Confidence level of doctors for diagnosing common adult and pediatric MSK pathologies was $55.4 \%$ and $33 \%$ respectively. Most of the doctors indicated that they were dissatisfied with their MSK education $(71 \%)$ as there was inadequate undergraduate teaching $(21.5 \%, 46.3 \%$ agreed to teaching for GALS, REMS) (Table-I).

Awareness of REMS was high amongst doctors (72/121 [59.5\%]), though less were aware of GALS technique (54/121 [44.6\%]) (Fig.1). Concomitantly, more doctors reported using REMS (69/121 [57 \%]) than GALS (50/121 [41.3\%]) in their clinical practice (Fig.2). Of those doctors who used GALS, most felt it had improved their ability to detect significant MSK pathology $(92 \%)$.

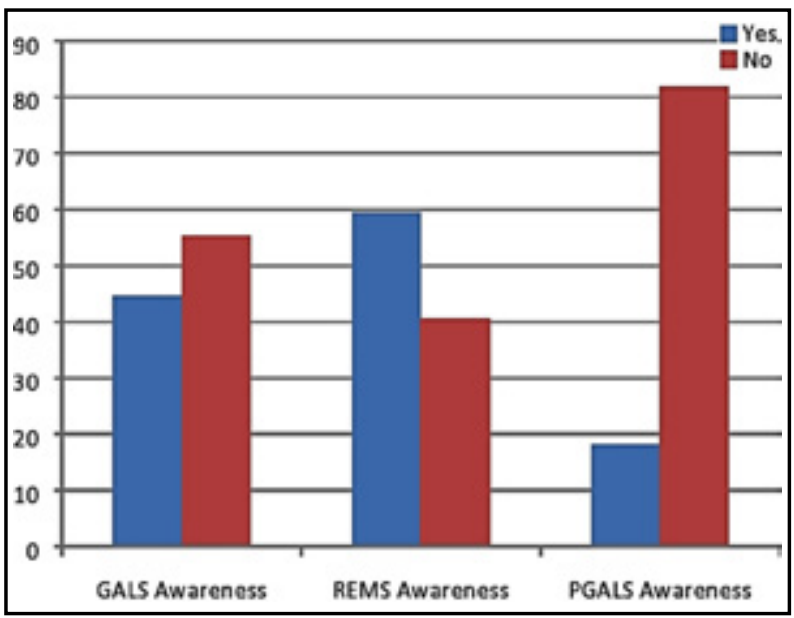

Fig.1: Percent Awareness of GALS/ REMS/pGALS among trainee doctors and non-specialists.
Undergraduate exposure to pediatric MSK medicine appeared to be low in the respondents (5.8\%), and so was its awareness (18.2\%). Almost $62 \%$ of trainees reported clinical contact with children on at least a weekly basis. Of these trainees, only $16 \%$ use pGALS in their current clinical practice, all of whom were general pediatric trainees. There was significant correlation of MSK Examinations awareness with undergraduate teaching and doctor's clinical experience (Table-II).

\section{DISCUSSION}

Musculoskeletal complaints constitute approximately $20 \%$ of visits to family physicians. ${ }^{13}$ The MSK examination is best taught in a handson, longitudinal fashion. Near-peer teaching refers to physicians-in-training teaching their junior colleagues. ${ }^{14}$ Lack of trained faculty leads to inadequate supervision of the trainees. ${ }^{15}$

In this survey we examined the awareness and use of structured approaches to MSK examination within the doctors in their current clinical practice. In a similar study done on trainee doctors it was found that awareness of GALS was more $91 \%$ as compared to REMS $21 \%$ while we found out that $44.6 \%$ were aware of GALS, $59.5 \%$ of REMS and $18.18 \%$ of pGALS. ${ }^{16}$

The majority of doctors rated GALS technique as simple to use and easy to remember. The GALS examination is sufficiently detailed to detect MSK pathologies. Beattie KA and colleagues found out that it may be a useful tool for physiotherapists to rule out rheumatoid arthritis. The sensitivity and specificity of GALS were 71 to

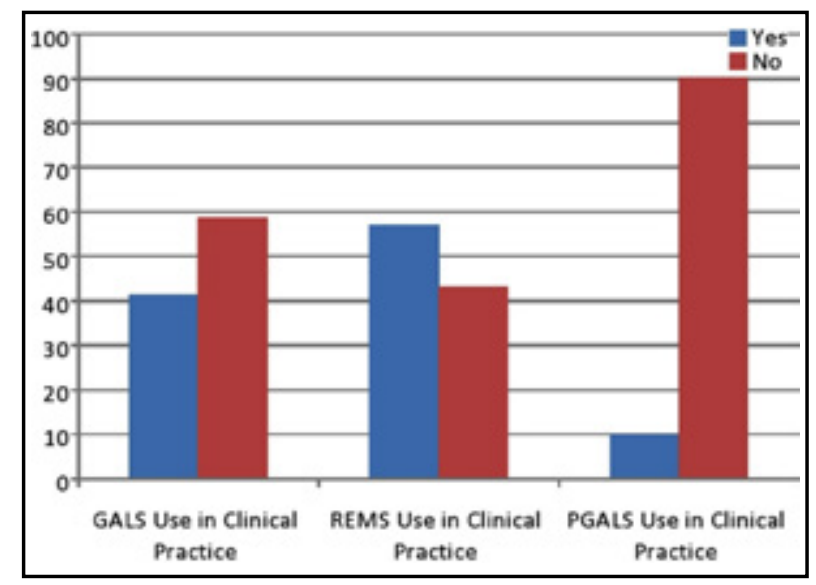

Fig.2: Percentage of use of GALS/REMS/pGALS among trainee doctors and non-specialist in clinical practice. 
Saba Saif et al.

Table-II: Comparison of GALS, REMS, PGALS with undergraduate teaching and clinical experience.

\begin{tabular}{|c|c|c|c|c|c|c|}
\hline & & \multicolumn{4}{|c|}{ Undergraduate Teaching } & \multirow[t]{2}{*}{$p$-value } \\
\hline & & & & & & \\
\hline \multirow[t]{2}{*}{ GALS Awareness } & Yes & 26 & $48 \%$ & 28 & $51.8 \%$ & $0.000^{*}$ \\
\hline & No & 0 & $0 \%$ & 67 & $100 \%$ & \\
\hline \multirow[t]{2}{*}{ REMS Awareness } & Yes & 21 & $29 \%$ & 51 & $70.8 \%$ & $0.013^{*}$ \\
\hline & No & 5 & $10.2 \%$ & 44 & $89.8 \%$ & \\
\hline \multirow[t]{4}{*}{ pGALS Awareness } & Yes & 6 & $27.27 \%$ & 16 & $72.72 \%$ & 0.465 \\
\hline & No & 20 & $20.2 \%$ & 79 & $79.8 \%$ & \\
\hline & & \multicolumn{4}{|c|}{ Clinical Experience } & p-value \\
\hline & & \multicolumn{2}{|c|}{$\begin{array}{c}<5 y r s \\
N 74 \% \\
\end{array}$} & \multicolumn{2}{|c|}{$\begin{array}{c}>5 y r s \\
N 47 \% \\
\end{array}$} & \\
\hline \multirow[t]{2}{*}{ GALS Awareness } & Yes & 23 & $42.59 \%$ & 31 & $57.4 \%$ & $0.000^{*}$ \\
\hline & No & 51 & $76.11 \%$ & 16 & $23.88 \%$ & \\
\hline \multirow[t]{2}{*}{ REMS Awareness } & Yes & 38 & $52.77 \%$ & 34 & $47.22 \%$ & $0.022^{*}$ \\
\hline & No & 36 & $73.46 \%$ & 13 & $26.53 \%$ & \\
\hline \multirow[t]{2}{*}{ pGALS Awareness } & Yes & 6 & $27.27 \%$ & 16 & $72.72 \%$ & $0.000^{*}$ \\
\hline & No & 68 & $68.68 \%$ & 31 & $31.31 \%$ & \\
\hline
\end{tabular}

*P value $<0.05$

$86 \%$ and 69 to $93 \%$, respectively. ${ }^{17}$ Another survey reported that following a very short training period, family physicians and nurse practitioners were able to use the GALS examination as a screening tool for RA. ${ }^{18}$

In our study most doctors were not confident in recognizing common MSK problems. In a similar study more than $30 \%$ could not confidently diagnose common rheumatic conditions, while $75 \%$ felt unable to diagnose a connective tissue disorder ${ }^{19}$ compared to $44.6 \%$ in our study. Similarly, $33 \%$ of our doctors could confidently diagnose pediatric MSK pathologies. Most doctors felt competent in hand assessment (68.8\%) as compared to foot examination. ${ }^{19}$

Undergraduate teaching for MSK examination is lacking in most medical schools. The American Association of Medical Colleges claims that most medical schools do not effectively educate future physicians on MSK medicine. ${ }^{20}$ According to our survey $21.5 \%, 46.3 \%$ and $5.8 \%$ agreed to teaching for GALS, REMS and pGALS respectively. PAL is a useful adjunct to MSK clinical skills training. Trainee confidence increased from 3.7 to 89.9 (P $<0.0001$ ) after PAL training. ${ }^{21}$ Jandial $S$ found that doctors lacked confidence in pediatric MSK clinical skills and many had little exposure to pGALS teaching due to time constraints and a lack of tutors. ${ }^{22}$
Most doctors showed dis-satisfaction with their MSK education. Al-Nammari SS found out that only $13 \%$ doctors felt they had adequate exposure to MSK medicine after completion of their foundation programmed as compared to $29 \%$ in our study. ${ }^{23}$ similarly in another study $80 \%$ of doctors felt that they had not received adequate teaching in MSK assessment. ${ }^{19}$

This study included a survey of MSK medicine practice at a local level including doctors from a broad range of specialties belonging to different medical colleges.

Limitations of the study: First it was a single center study. Secondly a bias may have affected our result i.e. responding trainee doctors may differ from the non-responders in their interest and exposure to MSK medicine. Thirdly, junior doctor's performance in clinical practice may not be comparable to their self-reported use and confidence in MSK examination routines.

Strength of the study: Wider studies including multiple institutes will bring strength to our observations and thus request can be made to review the national undergraduate and postgraduate medical curriculum. The quality and quantity of exposure to MSK medicine during the foundation programme must be improved by near-peer teaching. 


\section{CONCLUSION}

Major delay occurs in diagnosing MSK diseases due to deficiencies in MSK examination skills among physicians in training and non-specialists.

\section{Grant Support \& Financial Disclosures: None.}

\section{Declaration of conflicting Interests: None.}

\section{REFERENCES}

1. Lewis R, Constanza B, Gomez A, Rayman M, Lanham-New $\mathrm{S}$, Woolf A, et al. Strategies for optimizing musculoskeletal health in the $21^{\text {st }}$ century. BMC Musculoskelet Disord. 2019;20:164. doi: 10.1186/s12891-019-2510-7

2. Almoallim $\mathrm{H}$, Attar S, Jannoudi N, Al-Nakshabandi N, Eldeek B, Fathaddien O, et al. Sensitivity of Standardized Musculoskeletal Examination of the Hand and Wrist Joints in Detecting Arthritis in Comparison to Ultrasound Findings in Patients Attending Rheumatology Clinics. Clin Rheumatol. 2012;31(9):1309-1317. doi: 10.1007/s10067-0122013-5

3. Humaira, Maqboob A, Batool A, Hannan MA, Latif A. Frequency and Morbidity of Joint Pain above 40 years of Age. Pak J Med Health Sci. 2018;12(2):748-749.

4. Hawkn GA. The assessment of musculoskeletal pain. Clin Exp Rheumatol. 2017;35 Suppl 107(5):8-12.

5. BeattieKA,BobbaR,BayoumiI,ChanD,SchabortI,BoulosP, et al. Validation of the GALS musculoskeletal screening exam for use in primary care: a pilot study. BMC Musculoskelet Disord. 2008;9:115. doi: 10.1186/1471-2474-9-115

6. Patel V, Patel P, Jeffery R, Taylor J, Thomas H. Examination of the musculoskeletal system: junior doctors' perceptions of the usefulness of the Gait, Arms, Legs and Spine (GALS) technique. Postgrad Med J. 2015;91(1078):418-422. doi: 10.1136/ postgradmedj-2015-133340

7. Blake T. Teaching musculoskeletal examination skills to UK medical students: A comparative survey of Rheumatology and Orthopedic education practice. BMC Med Educ 2014;14:62. doi: 0.1186/1472-6920-14-62

8. Perry ME, Burke JM, Friel L, Field M. Can training in musculoskeletal examination skills be effectively delivered by undergraduate students as part of the standard curriculum? Rheumatology (Oxford, England). 2010;49(9):1756-1761. doi: 10 1093/rheumatology/keg 166

9. Foster HE, Jandial S. pGALS -A screening examination of the musculoskeletal system in school-aged children. Hands On. 2008;(15):1-8.

10. Foster HE, Kay LJ, Friswell M, Coady D, Myers A. Musculoskeletal screening examination (pGALS) for school-aged children based on the adult GALS screen. Arthritis Rheum. 2006;55(5):709-716.

11. Boulter EL, Rogers JR, Borland ML. Improving junior doctor's confidence in paediatric musculoskeletal assessment. J Paediatr Child Health. 2014;50(10):787-90. doi: $10.1111 /$ jpc. 12644

12. Siddharthan T, Soares S, Wang HH, Holt SR. Objective Structured Clinical Examination-Based Teaching of the Musculoskeletal Examination. South Med J. 2017;110(12):761-764. doi: 10.14423/SMJ.0000000000000739
13. Almoallim H. Determining and Prioritizing Competencies in the Undergraduate Internal Medicine Curriculum in Saudi Arabia. East Mediterr Health J. 2011;17:656-662.

14. Rosenberg CJ, Nanos KN, Newcomer KL. The "NearPeer" Approach to Teaching Musculoskeletal Physical Examination Skills Benefits Residents and Medical Students. PM R. 2017;9:251-257. doi: 10.1016/j. pmrj.2016.06.004

15. John S G Biggs. Postgraduate medical training in Pakistan: observations and recommendations. J Coll Physicians Surg Pak. 2008;18(1):58-63.

16. Baker KF, Jandial S, Thompson B, Walker D, Taylor $\mathrm{K}$, Foster HE. Use of structured musculoskeletal examination routines in undergraduate medical education and postgraduate clinical practice a UK survey. BMC Med Educ. 2016;16(1):277. doi: 10.1186/s12909-016-0799-6

17. Beattie KA, Macintyre NJ, Pierobon J, Coombs J, Horobetz D, Petric A, et al. The sensitivity, specificity and reliability of the GALS (gait, arms, legs and spine) examination when used by physiotherapists and physiotherapy students to detect rheumatoid arthritis. Physiotherapy. 2011;97(3):196202. doi: $10.1016 /$ j.physio.2010.11.008

18. Beattie KA, MacIntyre NJ, Cividino A. Screening for signs and symptoms of rheumatoid arthritis by family physicians and nurse practitioners using the Gait, Arms, Legs, and Spine musculoskeletal examination. Arthritis Care Res (Hoboken). 2012;64(12):1923-1927. doi: 10.1002/ acr. 21740

19. McCarthy EM, Sheane BJ, Cunnane G. Greater focus on clinical rheumatology is required for training in internal medicine. Clin Rheumatol. 2009;28:139-43. doi: 10.1007/s10067-008-0997-7

20. Day CS, YEh AC, Franko O, Ramirez M and Krupat E. Musculoskeletal Medicine: An Assessemnt of the Attituide and Knoweledge of Medical Studnets at Harverd Medical School. Acad Med. 2007;82:452-457.

21. Graham K, Burke JM, Field M. Undergraduate rheumatology: can peer-assisted learning by medical students deliver equivalent training to that provided by specialist staff? Rheumatology (Oxford). 2008;47(5):652655. doi: 10.1093/rheumatology/ken048

22. Jandial S, Stewart J, Foster HE. What do they need to know: achieving consensus on paediatric musculoskeletal content for medical students? BMC Med Educ. 2015;15:171. doi: 10.1186/s12909-015-0449-4

23. Al-Nammari SS, James BK, Ramachandran M. The inadequacy of musculoskeletal knowledge after foundation training in the United Kingdom. J Bone Joint Surg Bt. 2009;91(11):1413-1418. doi: 10.1302/0301-620X.91B11.22445

\section{Authors Contribution:}

SS: Conceived, designed and did manuscript writing and responsible for integrity of the article. SS, HM \& SF: Did data collection and statistical analysis.

SS: Did review and final approval of manuscript. 